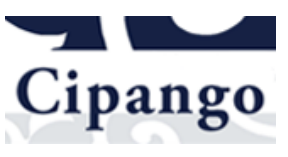

\title{
Cipango
}

Cahiers d'études japonaises

16 | 2009

L'invention des « arts populaires » - Yanagi Sōetsu et le Mingei

\section{Yanagi Sōetsu et l'invention des « arts populaires $»$ : remise en perspective}

Yanagi Sōetsu and the invention of 'folk crafts': A new contextualisation

\section{Christophe Marquet}

\section{(2) OpenEdition}

\section{Journals}

Édition électronique

URL : https://journals.openedition.org/cipango/371

DOI : $10.4000 /$ cipango.371

ISSN : 2260-7706

Éditeur

INALCO

Édition imprimée

Date de publication : 1 janvier 2009

Pagination : 13-22

ISSN : 1164-5857

Référence électronique

Christophe Marquet, "Yanagi Sōetsu et l'invention des « arts populaires » : remise en perspective », Cipango [En ligne], 16 | 2009, mis en ligne le 15 novembre 2011, consulté le 30 juin 2021. URL : http:// journals.openedition.org/cipango/371 ; DOI : https://doi.org/10.4000/cipango.371

Ce document a été généré automatiquement le 30 juin 2021.

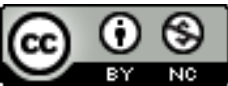

Cipango est mis à disposition selon les termes de la Licence Creative Commons Attribution - Pas d'Utilisation Commerciale 4.0 International. 


\title{
Yanagi Sōetsu et l'invention des « arts populaires » : remise en perspective
}

\author{
Yanagi Sōetsu and the invention of 'folk crafts': A new contextualisation
}

\author{
Christophe Marquet
}

1 L'exposition "L'esprit mingei au Japon $»^{1}$, conçue par Germain Viatte avec la collaboration de Shiraha Akemi pour le Musée du quai Branly, a permis de révéler en France au grand public un pan encore largement méconnu de l'art japonais, ainsi qu'un aspect de l'œuvre de Yanagi Muneyoshi (ou Sōetsu de son nom d'écrivain) 柳宗悦 (1889-1961). Elle fut certainement, à ce titre, l'une des plus intéressantes manifestations artistiques consacrées au Japon organisées à Paris en 2008.

2 L'approche choisie par les commissaires de cette exposition - annoncée dans le soustitre «de l'artisanat populaire au design » - visait à démontrer la continuité entre le mouvement historique des Arts populaires (mingei undō 民藝運動), lancé dans les années 1920, et la création contemporaine. Une large partie était en effet consacrée au designer Yanagi Sōri 柳宗理 (né en 1915)² , le fils aîné de Yanagi, et aux rencontres et collaborations internationales de ce mouvement, entre les années 1930 et 1950, avec des créateurs comme Bruno Taut, Charlotte Perriand ou Isamu Noguchi.

3 La présence d'une autre exposition consacrée au design japonais, Wa: l'harmonie au quotidien, à quelques centaines de mètres du Musée du quai Branly, à la Maison de la culture du Japon à Paris, évoquait, elle aussi Yanagi et venait encore renforcer ce sentiment qu'il avait été en définitive principalement l'initiateur d'un mouvement de redécouverte de l'artisanat japonais qui contribua à la revitalisation du design industriel moderne. Dans le catalogue de cette dernière exposition, la position de Yanagi sur la coexistence nécessaire de l'artisanat et du design industriel était, d'ailleurs présentée comme «le modèle premier de la vision qui anime le design d'aujourd'hui $»^{3}$. 
Cette interprétation a conduit les commissaires de l'exposition du Musée du quai Branly à minorer certains aspects importants du mouvement des Arts populaires, comme ses sources intellectuelles et esthétiques occidentales, son rapport au bouddhisme ou sa dimension picturale. Cette manifestation fut donc de nature assez différente des deux premières grandes expositions consacrées au Mingei organisées de 1995 à 1997 aux États-Unis à l'initiative de la Maison des arts populaires du Japon (Nihon mingeikan) $)^{4}$, et en 2000 au Musée des arts asiatiques de Nice, sur la base d'une collection privée ${ }^{5}$.

5 On assiste par ailleurs récemment à l'émergence de nouveaux types de lectures qui s'intéressent aux facteurs idéologiques à l'œuvre dans le travail de Yanagi. C'est notamment le cas de Kikuchi Yuko et de Kim Brandt, auteurs de deux ouvrages qui retracent l'histoire du mouvement Mingei dans le contexte du colonialisme, en y voyant avant tout une forme de «nationalisme culturel» ou de contribution à la formation d'un «style national moderne $»^{6}$. On trouvera une analyse du second de ces ouvrages à la fin de ce dossier. D'autres travaux, comme ceux d'Inaga Shigemi, ont cherché à mettre à jour le processus qui a conduit Yanagi à "inventer » une tradition esthétique asiatique dans le contexte du Japon colonial7

Certaines analyses plus radicales remettent en cause l'interprétation humaniste de l'œuvre de Yanagi et vont jusqu'à envisager des rapprochements entre certains aspects de son discours sur l'art populaire et l' " esthétique fasciste » des années 1930 et de la période de la Seconde Guerre mondiale en Europe ${ }^{8}$. La position de Yanagi à cette époque y est présentée comme celle d'un « moderniste réactionnaire »- expression que l'historien Jeffrey Herf avait appliquée aux régimes de l'Allemagne nazie et de l'Italie fasciste ${ }^{9}$ - qui rejeta l'individualisme de la société moderne et critiqua le caractère «maladif » de la création contemporaine.

7 Le présent dossier, sans ignorer ce type de lecture, vise surtout à apporter des éclairages sur des aspects de ce mouvement des Arts populaires qui n'ont pas été mis en avant par l'exposition du Musée du quai Branly, afin de mettre en perspective l'action de Yanagi dans ce domaine. Il est le fruit d'une rencontre consacrée à Yanagi Sōetsu, organisée le 15 janvier 2009 à l'InALco dans le cadre du séminaire du Centre d'Études Japonaises, en présence de Germain Viatte et de Shiraha Akemi.

Ce dossier reprend la majorité des interventions faites lors de cette journée, auxquelles ont été joints deux articles et une traduction qui portent sur des questions connexes. Avant de les présenter, rappelons brièvement la nature et les enjeux de ce mouvement Mingei initié par Yanagi.

\section{Yanagi et le mouvement des Arts populaires}

Yanagi Sōetsu occupe une place singulière dans le paysage intellectuel et artistique japonais de la première moitié $\mathrm{du} \mathrm{xx}^{\mathrm{e}}$ siècle : ni artiste, ni historien d'art au sens académique, on le qualifie volontiers de "penseur» versé dans les questions d'esthétique et de philosophie religieuse. Il fut surtout l'initiateur et le porte-parole du 《mouvement des Arts populaires» ou mingei undō. Ce terme de mingei 民藝 fut forgé en 1925 par Yanagi et deux céramistes, Kawai Kanjirō et Hamada Shōji, qui entendaient réhabiliter la «beauté fonctionnelle » de la poterie paysanne produite par des artisans anonymes. 
10 Il a été compris diversement au sens d'《 art folklorique» (minzoku geijutsu 民俗藝術), d'《art paysan » (nōmin bijutsu 農民美術) ou encore d'« art populaire » (minshū geijutsu 民衆藝術). Yanagi a récusé ces interprétations ${ }^{10}$, refusant en particulier l'usage du terme "art» (bijutsu, geijutsu), trop marqué par une définition issue de catégories artistiques occidentales importées au Japon à l'époque de Meiji et qui, à ses yeux, ont faussé le regard porté sur les objets ordinaires. Yanagi entendait ce néologisme au sens d'«artisanat » (gei) du «peuple» ( $\mathrm{min})$. Il prôna d'ailleurs aussi l'usage d'autres mots moins ambigus et plus concrets, comme getemono 下手物 (« objet commun ») - terme argotique utilisé par les brocanteurs sur les marchés aux puces qu'il fréquentait à Kyōto dans les années 1920 - de minki 民器 (《objet populaire») ou encore de zakki 雑器 (" objet courant»), qui n'ont toutefois pas connu la même fortune ${ }^{11}$.

11 Concrètement, ce terme mingei désignait de manière générique toutes sortes d'objets et d'ustensiles domestiques, du mobilier ou encore des vêtements et des textiles issus d'un artisanat manuel disparu ou en voie de disparition au début $\mathrm{du} \mathrm{xx}^{\mathrm{e}}$ siècle. Il s'agissait surtout de productions provinciales de la fin de l'époque d'Edo, voire du début de Meiji, même si des objets beaucoup plus anciens, comme des sculptures en pierre de l'époque Jōmon, retiendront plus tard l'attention de Yanagi pour leur primitivisme.

12 Yanagi a défini très tôt les critères de ce mingei, qui sont principalement la création anonyme, la fonction utilitaire, la multiplicité, le bas prix, la sobriété et la destination populaire, par opposition à l'artisanat d'art ou à l'œuvre d'art signée par un créateur, unique et luxueuse, produite pour les classes supérieures.

13 À première vue, cette définition qui accorde une place centrale à l'« ustensilité » de l'objet n'est guère éloignée de celle que les spécialistes des études folkloriques ont pu donner de l'art populaire dans d'autres pays, à commencer par la France. Que l'on pense aux travaux de Georges Henri Rivière ${ }^{12}$ au Musée des Arts et Traditions populaires à partir de la fin des années 1930 et à ceux de son successeur Jean Cuisenier par exemple ${ }^{13}$. Rappelons d'ailleurs que dès 1928 se constitua à Prague une Commission internationale des arts populaires ${ }^{14}$ sous l'égide de la Société des Nations, à laquelle prit part le Japon, avec l'objectif de "dresser le répertoire de leurs survivances traditionnelles", mais aussi d'envisager "les moyens de maintenir en vie ceux qui existaient encore ».

Mais Yanagi a bien précisé ce qui distinguait son projet de celui des musées d'ethnographie, comme le Nordiska Museum de Stockholm qu'il visita en 1929 lors de son voyage en $\operatorname{Europe~}^{15}$. Là où son initiative se démarque de l'approche ethnographique, c'est qu'elle ne visait pas à collecter en grand nombre des « œuvres de référence » à titre de témoignage d'une pratique, d'une histoire ou d'un milieu, mais qu'elle consistait à opérer une sélection d'abord qualitative selon des critères esthétiques subjectifs. La qualité plastique de l'objet étant néanmoins envisagée du point de vue de son usage, ce qui a donné naissance à la célèbre formule yō no bi 用の美, la «beauté fonctionnelle ».

15 En outre, l'un des principaux objectifs des collectes de Yanagi et des partisans de son mouvement était de proposer les pièces retenues comme des modèles pour la création d'un nouvel artisanat d'art, voire d'une nouvelle architecture et même d'une nouvelle forme d'art pictural figuratif ${ }^{16}$. Ces deux derniers aspects sont probablement les moins connus, car leur succès fut moindre. 

catalysa autour d'un projet muséographique, formulé dès 1926 et concrétisé en 1936 avec l'ouverture à Tōkyō de la Maison des arts populaires du Japon. Celui-ci était sous-tendu avant tout par un discours esthétique et non par une approche méthodique et scientifique, même si le choix des objets fut soumis à un ensemble de critères sélectifs. Il faut souligner le caractère pionnier de cette entreprise menée grâce à des fonds privés, à une époque où les musées d'État se désintéressaient de l'artisanat populaire, car il ne relevait pas de la démarche de construction identitaire fondée sur les «arts nobles» promue par ces institutions. Yanagi a rappelé en effet que la proposition qu'il avait faite à la fin des années vingt au Musée impérial de Tōkyō de donner ses collections pour qu'elles y soient présentées, resta sans répons $\mathrm{e}^{17}$.

17 Pour faire partie de la catégorie mingei, les objets devaient posséder pour Yanagi certaines qualités, à commencer par «l'honnêteté envers la finalité d'usage ». Ceci excluait les produits dont la fabrication était mécanisée ou qui étaient inutilement ornementés. Il s'agissait en partie d'une réaction au déclin de l'artisanat et à la normalisation industrielle des produits d'usage courant depuis l'époque de Meiji ${ }^{18}$.

int si l'objet mingei, selon Yanagi, ne devait pas être destiné à la contemplation esthétique, mais avoir cette valeur d'usage, il ne se résumait pas uniquement à une vocation purement fonctionnaliste. Il devait répondre aussi à un critère de "beauté ", ce qui ne fut pas d'ailleurs sans introduire une certaine ambiguïté dans ce projet. Se pose donc ici, un peu comme dans le cas des "arts premiers", la question de l'autonomie de l'objet, de la reconnaissance de sa valeur artistique au-delà de sa valeur d'usage. Par ailleurs, l'implication dans ce mouvement de créateurs aux productions souvent coûteuses - dont certains deviendront après la guerre des «trésors nationaux vivants »- est l'une de ses nombreuses contradictions.

19 La formation de ce terme emblématique mingei visa à englober un mouvement original qui fut à la fois une relecture, une redécouverte de l'artisanat du passé et une promotion de la création contemporaine, l'un enrichissant l'autre. Ce mouvement d'invention d'une tradition artisanale peut être également considéré au regard de sa contribution à la réévaluation de l'histoire de l'art japonais, en prenant en compte ses formes les plus populaires.

Le mouvement des Arts populaires poursuit encore ses activités aujourd'hui grâce à un réseau d'associations régionales réparties à travers tout le Japon et d'une trentaine de musées affiliés, réunis sous la bannière de l'Association des arts populaires du Japon (Nihon mingei kyōkai 日本民藝協会). Notons aussi qu'il a bénéficié d'une reconnaissance internationale, puisque le concept a été en quelque sorte exporté, notamment aux États-Unis. Il faut citer à cet égard l'expérience menée par le Mingei International Museum fondé à San Diego (Californie) en 1978 par Martha Longenecker ${ }^{19}$, céramiste et professeur d'art à l'Université de San Diego, qui avait notamment travaillé au Japon avec Hamada Shōji et connu Yanagi. Cette fondation publique se donne pour objectif, en appliquant les idées des Arts populaires, de " réévaluer la beauté d'usage dans les arts folkloriques, l'artisanat et le design de toutes les aires et les cultures du monde " et organise de nombreuses expositions de qualité dans ce domaine. 


\section{Contributions du dossier}

21 Le mouvement des Arts populaires peut être analysé selon des perspectives variées: son apport au design moderne - c'est l'approche privilégiée par l'exposition du Musée du quai Branly -, sa relecture de l'art japonais (en particulier de l'époque d'Edo), sa dimension esthétique, voire sociale. Il pose en outre de nombreuses questions, comme son rapport à l'ethnologie ou sa position à l'égard de la question coloniale. Ce dossier ne saurait prétendre répondre à toutes ces interrogations, mais il tente d'apporter quelques éclairages sur des points qui ont fait débat ces derniers temps.

Michael Lucken revient sur l'importante question des sources intellectuelles et esthétiques du mouvement des Arts populaires. Car si en apparence on peut parler de revirement dans les centres d'intérêt de Yanagi au milieu des années 1910, au moment où il commence à s'intéresser à l'art populaire coréen, ses références restent en majorité occidentales. On a souvent présenté le Mingei comme un mouvement antimoderniste, comme une antithèse à la conception occidentale de l'art individualiste. Mais on ne peut oublier que ce mouvement de relecture de la tradition artistique japonaise repose en partie sur des idées puisées chez des penseurs européens de la fin du XIX ${ }^{e}$ siècle, tel William Morris qui initia le mouvement Art and Crafts, et sur des thèmes qui furent véhiculés par des revues artistiques comme The Studio ${ }^{20}$.

L'attention portée par Yanagi à différentes formes d'arts figuratifs populaires - depuis la peinture coréenne jusqu'aux images d'ōtsu, en passant par les plaquettes votives -, est abordée par Christophe Marquet. Ce domaine peut sembler a priori le plus étranger aux critères d'ustensilité et d'anonymat définis par le mouvement des Arts populaires. L'intérêt de l'approche de Yanagi a été justement de révéler un ensemble d'œuvres négligées par les recherches académiques, car relevant de "l'imagerie populaire», et d'en analyser la nature, la fonction et l'histoire.

François Macé aborde la contribution essentielle de Yanagi à la redécouverte au début des années 1920 d'un moine sculpteur du xvIII ${ }^{\mathrm{e}}$ siècle, Mokujiki 木喰 (1718-1810), jusqu'alors totalement ignoré par les historiens de l'art bouddhique, car ses œuvres "ne répondaient pas aux critères du beau ». Cette découverte, qui précède de peu la formation du concept de mingei, révèle la nature de la méthode d'enquête de Yanagi et surtout la qualité et la pertinence de son regard, capable de "saisir une nouvelle beauté à partir de la laideur », une beauté qui ne s'exprime pas par le raffinement de la technique.

Enfin, le débat entre Yanagi Sōetsu et le grand folkloriste Yanagita Kunio 柳田國男 (1875-1962), au début des années 1940, est examiné en détail par Damien Kunik. Il permet de mieux comprendre les différences méthodologiques et idéologiques entre ces deux mouvements contemporains que sont les études folkloriques et les Arts populaires, qui à cette époque étaient parfois confondus, sans doute par le fait qu'ils s'intéressaient en partie au même type d'objets. On comprend parallèlement combien l'un et l'autre ont pu participer, dans ces années qui encadrent la Seconde Guerre mondiale, à cette réévaluation de l'apport du peuple $(\mathrm{min})$ à la culture japonaise dans son ensemble. Une traduction de l'unique entretien public entre Yanagi et Yanagita (diffusé sur les ondes radiophoniques en 1940) est également proposée par Kunik avec la contribution de Jean-Michel Butel, permettant de juger de la nature du débat entre 
les deux intellectuels et de leur positionnement respectif vis-à-vis de matériaux communs à leurs champs d'investigation.

Ce texte nous laisse penser que le folkloriste Yanagita se plaça du côté de la science descriptive et refusa d'intervenir dans le débat politique (tout au moins du point de vue de ses recherches), son mouvement n'entendant pas «agir sur la culture ». La réalité est en vérité plus complexe, même si l'on peut voir, dans cette position de principe, un point de différence entre les deux disciplines. Yanagi, sans être dénué d'ambition scientifique, envisagea le mingei avant tout comme un moyen de fixer des normes esthétiques. Son action supposait des jugements de valeur sur les objets, des choix, alors que l'approche des folkloristes et des archéologues visait à établir des constats à partir de l'étude des matériaux.

Pour clore ce dossier, on trouvera une analyse critique par Coralie Castel de trois expositions d'art japonais organisées à Paris en 2008 - y compris celle du Musée du quai Branly -, centrée sur le discours et les représentations qu'elles véhiculent à propos de «l'identité japonaise ».

La bibliographie des quatre premiers articles, qui se répondent l'un l'autre, a été rassemblée en fin de dossier afin qu'elle puisse servir à des études ultérieures.

\section{NOTES}

1. Germain Viatte (dir.), L'esprit mingei au Japon, Actes Sud, Musée du quai Branly, 2008. Catalogue de l'exposition «L'esprit mingei au Japon, de l'artisanat populaire au design », présentée dans la galerie du jardin du Musée du quai Branly du 30 septembre 2008 au 11 janvier 2009.

2. Après avoir étudié la peinture à l'occidentale à l'École des beaux-arts de Tōkyō, Yanagi Sōri devint l'assistant de Charlotte Perriand de 1940 à 1942. En 1950, il fonda l'Atelier Yanagi de recherche sur le design industriel (Yanagi indasutoriaru dezain kenkyūjo), au sein duquel il créa de nombreux objets usuels en s'appuyant notamment sur les savoir-faire traditionnels et en s'inscrivant dans l'esprit du mingei. À partir de 1977, il prit la direction de la Maison des arts populaires (Mingeikan) fondée à Tōkyō par son père. Voir le catalogue de l'exposition Yanagi Sōri. Seikatsu no naka no dezain 柳宗理—生活のなかのデザイン (Yanagi Sōri. Le design dans la vie quotidienne), Tōkyō kokuritsu kindai bijutsukan, 2007 et en particulier l'essai de Kida Takuya 木田拓也, 《Yanagi Sōri no dezain to mingei » 柳宗理のデザインと民藝 (Le design de Yanagi Sōri et le mingei), p. 12-14.

3. Fukagawa Masafumi, «Visages du “Wa". Harmonie dans le design de produits au Japon », in Kashiwagi Hiroshi et al., Wa : l'harmonie au quotidien. Design japonais d'aujourd'hui, Paris, Maison de la culture du Japon, 2008, p. 23.

4. Mingei. Two Centuries of Japanese Folk Art (Peabody Essex Museum, Joslyn Art Museum, Asian Art Museum of San Francisco, Fort Worth Museum of Science and History), The Japan Folk Crafts Museum, 1995. Cette exposition visait à présenter les différents aspects de l'artisanat populaire japonais à travers un échantillon de 140 pièces datant $\mathrm{du} \mathrm{XvI}^{\mathrm{e}} \mathrm{au} \mathrm{xx}^{\mathrm{e}}$ siècle, classées selon leur matériau (textile, céramique, laque, bois, métal, peintures).

5. Marie-Pierre Foissy-Aufrère, Eliza Barrère, Dominique Buisson, Robert Moes, Mingei de la collection Montgomery. Beauté du quotidien au Japon, Nice, Musée des arts asiatiques, 2000. 
6. Kikuchi Yuko, Japanese Modernisation and Mingei Theory. Cultural Nationalism and Oriental Orientalism, New York, Routledge Curzon, 2004; Kim Brandt, Kingdom of Beauty. Mingei and the Politics of Folk Art in Imperial Japan, Durham, Duke University Press, 2007.

7. Inaga Shigemi, "Reconsidering the Mingei Undō as a Colonial Discourse: The Politics of Visualizing Asian 'Folk Craft”, Asiatische Studien, 1999, vol. 53, nº 2, pp. 219-230.

8. Aso Noriko, "Mediating the Masses: Yanagi Sōetsu and Fascism", in Alan Tansman et Marilyn Ivy (éd.), The Culture of Japanese Fascism, Durham, Duke University Press, 2009, p. 139-154; Alan Tansman, The Aesthetics of Japanese Fascism, Berkeley, University of California Press, 2009, et en particulier "A Vision of Beautiful Things: Yanagi Sōetsu”, pp. 107-118.

9. Jeffrey Herf, Reactionary Modernism: Technology, Culture, and Politics in Weimar and the Third Reich, Cambridge, Cambridge University Press, 1984.

10. Voir son texte 《 Mingei no shushi » 民藝の趣旨 (La nature du mingei), 1933, in Yanagi Sōetsu, Mingei yonjū-nen 民藝四十年 (Quarante années du mouvement des Arts populaires), Tōkyō, 1958, réédité par Iwanami shoten, coll. "Iwanami bunko », 1984, p. 159-173. Ce texte a été traduit par Anne Bayard-Sakai dans L'esprit mingei au Japon, op. cit., p. 13-24.

11. Sur ces questions terminologiques, voir l'analyse d'Élisabeth Frolet dans Yanagi Sōetsu ou les éléments d'une renaissance artistique au Japon, Paris, Publications de la Sorbonne, 1986, p. 74-76.

12. Voir Nina Gorgus, Le magicien des vitrines. Le muséologue Georges Henri Rivière, Paris, Éditions de la Maison des sciences de l'homme, 2003.

13. Voir notamment Jean Cuisenier, L'art populaire en France. Rayonnement, modèles et sources, Fribourg, Office du Livre, 1975.

14. Voir Nina Gorgus, "Georges Henri Rivière et l'Europe ", in Jacqueline Christophe, DenisMichel Boëll, Régis Meyran, Du folklore à l'ethnologie, Paris, Éditions de la Maison des sciences de l'homme, 2009, p. 370. Cette commission donnera naissance en 1964 à la Société internationale d'Ethnologie et de Folklore.

15. Voir Yanagi Sōetsu, «Mingeikan no seiritsu »民藝館の生立 (La création de la Maison des arts populaires), 1935, repris dans Yanagi Sōetsu zenshū 柳宗悦全集 (๕uuvres complètes de Yanagi Sōetsu, plus loin YSZ), t. 16, Tōkyō, Chikuma shobō, 1981, p. 52-53.

16. Notons que l'idée du « rôle artistique » du musée d'ethnographie, où les objets seraient des sources d'inspiration pour les artistes et la diffusion de techniques inconnues, n'était pas étrangère au projet d'un Georges Henri Rivière formulé en 1931. Voir Nina Gorgus, Le magicien des vitrines, op. cit., p. 55.

17. Voir Yanagi Sōetsu, "La beauté en quête de critères ", in Artisan et inconnu. La beauté dans l'esthétique japonaise, adapté par Bernard Leach, traduit par Mathilde Bellaigue, Paris, L'Asiathèque, 1992, p. 17.

18. Ibid., p. 19.

19. Martha Longenecker est notamment l'auteur de Mingei of Japan. The Legacy of the Founders. Sōetsu Yanagi, Shōji Yamada, Kanjirō Kawai, San Diego, Mingei International Museum, 2006.

20. Cette hybridité du mingei a été soulignée par Élisabeth Frolet dans «Yanagi Sōetsu et son mouvement d'art populaire, le mingei undō. Le rôle ambigu de l'Europe et de sa modernité dans la formation d'un mouvement artistique japonais dans les années 30 ", Gazette des Beaux-Arts, t. cIX, $1417^{\mathrm{e}}$ livraison, février 1987, p. 87-96. 


\section{RÉSUMÉS}

Introduction au dossier sur le mingei undō (mouvement des Arts populaires) et son fondateur, Yanagi Sōetsu, fruit d'une rencontre qui s'est tenue le 15 janvier 2009 à l'INALco.

Introduction to the issue on mingei undō (Folk Art movement) and its founder, Yanagi Sōetsu, which is the result of a symposium held at INALCO on January 15, 2009.

\section{INDEX}

Keywords : Utensils, Ethnology, Folk Art, Popular Culture, Mingei, Yanagi Sōetsu (1889-1961), Anthropology

Thèmes : anthropologie, ethnologie, histoire de l'art

キーワード : minshū geijutsu 民衆芸術, busshitsu bunka 物質文化, taishū bunka 大衆文化, minzoku 民俗, mingei undō 民芸運動, nichiyōhin 日用品, mingeihin 民芸品, Yanagi Sōetsu 柳宗 悦 (1889-1961), jinruigaku 人類学, minzokugaku 民族学, bijutsushi 美術史, Taishō jidai 大正時代 (1912-1923), Shōwa jidai 昭和時代 (1923-1945), Shōwa jidai 昭和時代 (1945-1989)

Mots-clés : arts populaires, culture matérielle, culture populaire, folklore, Yanagi Sōetsu (1889-1961), objets, objets usuels

Index chronologique : Shōwa (1923-1945), Shōwa (1945-1989) 